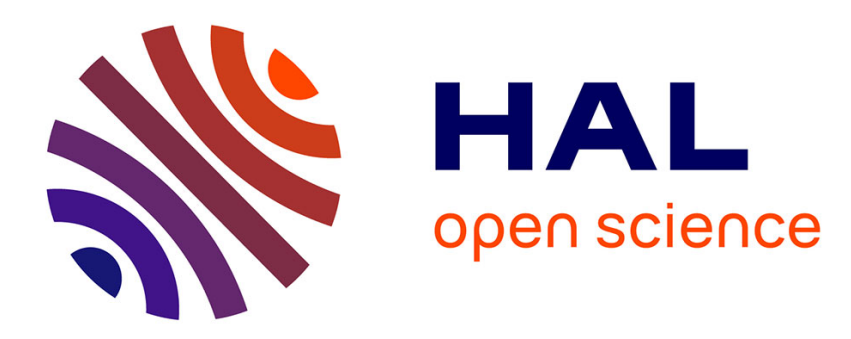

\title{
Etude par spectrométrie Mössbauer d'alliages Fe-Si-B amorphes
}

\author{
J.M. Dubois, M. Bastick, Gérard Le Caër, C. Tete
}

\section{To cite this version:}

J.M. Dubois, M. Bastick, Gérard Le Caër, C. Tete. Etude par spectrométrie Mössbauer d'alliages Fe-Si-B amorphes. Revue de Physique Appliquée, 1980, 15 (6), pp.1103-1111. 10.1051/rphysap:019800015060110300 . jpa-00244828

\section{HAL Id: jpa-00244828 https://hal.science/jpa-00244828}

Submitted on 1 Jan 1980

HAL is a multi-disciplinary open access archive for the deposit and dissemination of scientific research documents, whether they are published or not. The documents may come from teaching and research institutions in France or abroad, or from public or private research centers.
L'archive ouverte pluridisciplinaire HAL, est destinée au dépôt et à la diffusion de documents scientifiques de niveau recherche, publiés ou non, émanant des établissements d'enseignement et de recherche français ou étrangers, des laboratoires publics ou privés. 


\title{
Etude par spectrométrie Mössbauer d'alliages Fe-Si-B amorphes
}

\author{
J. M. Dubois, M. Bastick, G. Le Caer \\ Laboratoire de Métallurgie (*), L.A. 159, Ecole des Mines, 54042 Nancy Cedex, France \\ et C. Tete \\ Laboratoire de Physique du Solide $\left({ }^{*}\right)$, L.A. 155, Ecole des Mines, 54042 Nancy Cedex, France
}

(Reçu le 14 novembre 1979, révisé le 7 janvier 1980, accepté le 7 janvier 1980)

\begin{abstract}
Résumé. - Des alliages amorphes $\mathrm{Fe}_{100-x-y} \mathrm{Si}_{y} \mathrm{~B}_{x}$ ont été étudiés par spectrométrie Mössbauer. Les méthodes de calcul et les origines possibles des dissymétries des spectres sont discutées. La comparaison des distributions de champ hyperfin des alliages avec et sans silicium suggère que le silicium et le fer occupent des sites semblables (pour les valeurs de $y$ étudiées, $y \leqslant 9$ ) alors que le bore occupe une position interstitielle avec $\simeq 9$ atomes de fer proches voisins. Les alliages Fe-B sont fortement stabilisés par le silicium. Les étapes et les mécanismes de cristallisation d'alliages $\mathrm{Fe}_{82} \mathrm{Si}_{3} \mathrm{~B}_{15}$ et $\mathrm{Fe}_{76} \mathrm{Si}_{9} \mathrm{~B}_{15}$ ont été précisés. Ils diffèrent de ceux observés dans les alliages $\mathrm{Fe}-\mathrm{B}$ car il ne se forme pas de borures métastables.
\end{abstract}

\begin{abstract}
Amorphous $\mathrm{Fe}_{100-x-y} \mathrm{Si}_{y} \mathrm{~B}_{x}$ alloys have been studied by Mössbauer Spectroscopy. The fitting methods and some possible origins of the asymmetric broadenings of the peaks are discussed. A comparison of the hyperfine field distributions in alloys with and without silicon suggests that iron and silicon are located in similar sites (for the studied $y$ values, $y \leqslant 9$ ) while boron occupies interstitial sites with $\simeq 9$ iron atoms nearest neighbours. Crystallisation steps and mechanisms have been studied in $\mathrm{Fe}_{82} \mathrm{Si}_{3} \mathrm{~B}_{15}$ and $\mathrm{Fe}_{76} \mathrm{Si}_{9} \mathrm{~B}_{15}$ alloys. They differ from those found in Fe-B alloys because metastable borides are not observed.
\end{abstract}

1. Introduction. - De nombreux travaux ont été récemment consacrés aux alliages métalliques amorphes Fe-B. Ils ont permis de caractériser les propriétés mécaniques, magnétiques, la stabilité thermique et les mécanismes de cristallisation [1] de ces alliages.

L'étude de leur structure a été abordée soit à l'aide de modèles théoriques $[2,3,4]$, soit à partir de la diffraction des rayons $X$ [5] ou de la spectrométrie Mössbauer [6, 7, 8]. Les résultats d'EXAFS [9] et de diffraction des neutrons [10] seront bientôt disponibles. Les modèles théoriques diffèrent par la prise en compte ou non de l'ordre chimique local c'est-à-dire des interactions métal de transition-métalloïde. Dans le modèle de Polk, les atomes de bore se placent dans des interstices déterminés uniquement par l'empilement des atomes métalliques [2]. Les modèles quasi cristallins de Vincze et al. [11] et de Gaskell [4] font intervenir un polyèdre de coordination bien défini autour des métalloïdes : le prisme trigonal d'atomes métalliques centré par le métalloïde qui est l'unité

(*) Associé au C.N.R.S. de construction commune à de nombreux siliciures, borures, phosphures et carbures. Ces deux derniers modèles diffèrent par la façon d'empiler ces unités.

L'addition de silicium aux alliages Fe-B permet de diminuer, d'un ordre de grandeur, la vitesse critique de trempe qui conduit à l'état amorphe [12]. Cet élément augmente d'ailleurs la stabilité thermique de nombreux systèmes amorphes, métastables ou cristallisés tels la phase $\varepsilon$ Fe-C-Si ou le carbure $\varepsilon$ des aciers au silicium [13]. Le présent travail porte alors sur l'influence du silicium sur la structure amorphe étudiée par spectrométrie Mössbauer et sur les mécanismes de retour à l'équilibre d'alliages $\mathrm{Fe}_{100-x-y} \mathrm{Si}_{y} \mathrm{~B}_{x}$.

\section{Conditions expérimentales et domaine d'existence} de l'état amorphe. - Les alliages de base ont été obtenus par fusion d'alliages binaires $\mathrm{Fe}_{2} \mathrm{~B}, \mathrm{Fe}_{66} \mathrm{Si}_{34}$ et de fer au four à induction sur sole refroidie et sous atmosphère d'argon purifié. Cette procédure permet d'éliminer les pertes de métalloïde que l'on constate si l'on fond directement les trois constituants $\mathrm{Fe}, \mathrm{Si}$, B purs. Dans cette étude, nous avons utilisé du fer 
électrolytique préalablement dégazé par fusion sous vide, du bore de pureté 99,9 et du silicium 99,999.

Les lingots ayant subi une perte de masse supérieure à $0,3 \%$ ont été éliminés. Leur homogénéité a été contrôlée à l'aide d'une microsonde de Castaing. La composition des alliages amorphes a été déterminée à l'aide d'un analyseur ionique en utilisant comme étalons les rubans amorphes préparés à partir de lingots n'ayant subi aucune perte de masse lors de leur élaboration. Cette méthode suppose que la trempe depuis l'état liquide, à l'aide d'un appareil à disque semblable à celui décrit par Liebermann et Graham [14], n'introduit pas de changement de composition. Cette hypothèse est raisonnable car les alliages sont chauffés par induction et il n'y a pas de réaction entre le creuset de quartz et l'alliage liquide qui est maintenu sous atmosphère d'argon purifié. Le liquide est projeté sur la surface d'un disque de cuivre dont la vitesse périphérique est choisie entre 15 et $40 \mathrm{~ms}^{-1}$. La vitesse de trempe estimée èst de l'ordre de $10^{6} \mathrm{~K} \mathrm{~s}^{-1}$.

L'état amorphe des échantillons a été contrôlé par diffraction des rayons $X$. Une étude systématique d'alliages ternaires $\mathrm{Fe}-\mathrm{Si}$-B a permis de déterminer les limites du domaine d'existence de l'état amorphe. Nos résultats se comparent bien à ceux de Luborsky et al. [15] et de Fujimori et Masumoto [16] qui ont utilisé la même méthode de trempe.

Enfin, les spectres Mössbauer ont été enregistrés à $300 \mathrm{~K}$ à l'aide d'un spectromètre à accélération constante et d'une source de ${ }^{57} \mathrm{Co}$ diffusée dans une matrice de rhodium. Les déplacements isomériques sont donnés par rapport à celui du fer $\alpha$ à $300 \mathrm{~K}$. La largeur caractéristique des raies du fer $\alpha$ à $300 \mathrm{~K}$ sera notée $\Gamma$ et les six pics d'un spectre seront notés de 1 à 6 des vitesses négatives aux vitesses positives.

3. Paramètres hyperfins moyens. - Au contraire des distributions de champ hyperfin dont la structure fine dépend essentiellement de l'ordre chimique local [17], les paramètres moyens (champ hyperfin moyen $\vec{H}$, déplacement isomérique moyen $\delta$, intensité des transitions $\Delta m=0$ ) sont peu sensibles aux hypothèses de calcul des spectres.

A la température ambiante, les spectres comportent six raies élargies $\left(\Gamma_{1} \simeq 1,5 \mathrm{~mm} \mathrm{~s}^{-1}, \Gamma_{2} \simeq 1 \mathrm{~mm} \mathrm{~s}^{-1}\right.$, $\Gamma_{3} \simeq 0,5 \mathrm{~mm} \mathrm{~s}^{-1}$ ) qui résultent de l'existence d'une distribution de champ hyperfin. Ils peuvent être analysés à l'aide de six raies Lorentziennes par une méthode de moindres carrés. Les paramètres $\bar{H}$ et $\bar{\delta}$ déduits de ce calcul simple diffèrent peu de ceux qui sont déduits d'un calcul plus complexe des distributions de champ hyperfin $\left(\bar{H}_{\mathrm{D}}, \bar{\delta}_{\mathrm{D}}\right)$. On trouve

$$
\begin{gathered}
1 \leqslant\left|\bar{H}-\bar{H}_{\mathrm{D}}\right| \leqslant 4 \mathrm{kOe}, \\
0 \leqslant\left|\bar{\delta}-\bar{\delta}_{\mathrm{D}}\right| \leqslant 0,015 \mathrm{~mm} \mathrm{~s}^{-1} .
\end{gathered}
$$

La figure 1 présente l'évolution de $\bar{H}$ et $\bar{\delta}$ en fonction de la teneur en silicium pour des teneurs en bore

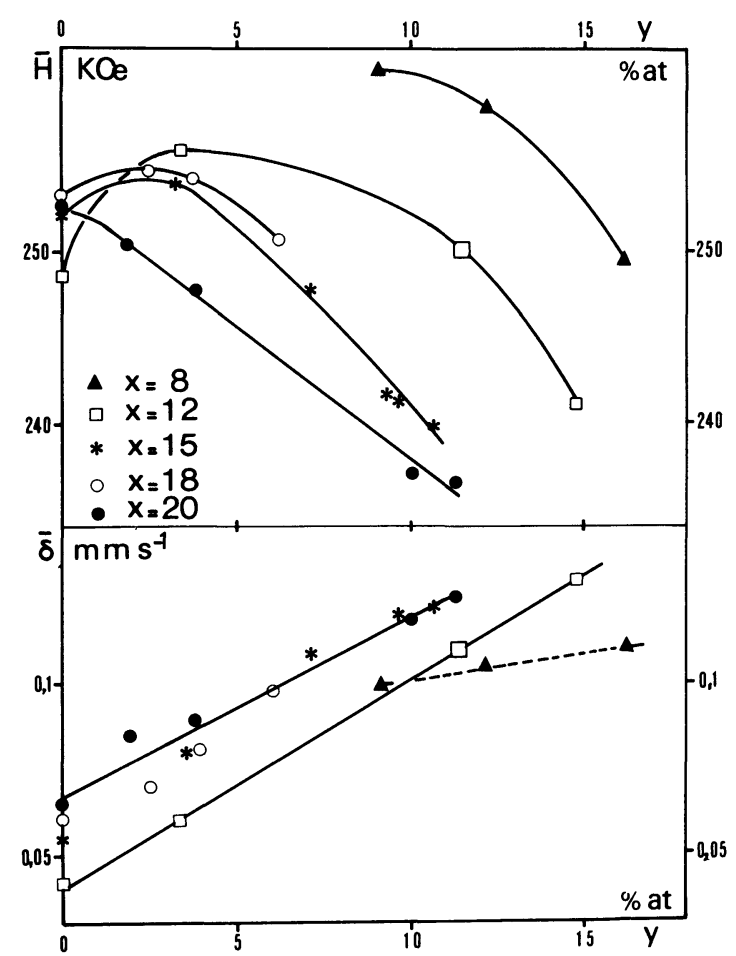

Fig. 1. - Champs hyperfins moyens $\bar{H}$ et déplacements isomériques moyens $\bar{\delta}$ à température ambiante en fonction de la teneur en silicium pour différentes teneurs en bore.

[Mean hyperfine fields $\bar{H}$ and mean isomer shifts $\bar{\delta}$ at room temperature as functions of silicon content for different boron contents.]

constantes $(x=8,11,5 \leqslant x \leqslant 13,15$ et 20 at. $\% \mathrm{~B})$. L'allure linéaire des courbes pour $y>3$ at. $\% \mathrm{Si}$ permet une vérification a posteriori des dosages effectués par émission ionique secondaire. Le rapport entre le champ hyperfin moyen et le moment à saturation à $300 \mathrm{~K}$ [15] est tel que $125 \leqslant \bar{H} / \bar{\mu} \leqslant 140 \mathrm{kOe} / \mu_{\mathrm{B}}$. Ces valeurs sont caractéristiques de nombreux alliages amorphes dérivés du système $\mathrm{Fe}-\mathrm{B}$ et sont très proches $\mathrm{du}$ rapport $\left(130 \mathrm{kOe} / \mu_{\mathrm{B}}\right)$ rencontré dans de nombreux composés : carbures, borures, phosphures. On observe toutefois que l'écart à ce rapport augmente avec la teneur en silicium.

4. Distributions de champ hyperfin. - Les distributions de champ hyperfin $H$ ont été calculées à l'aide d'une méthode de dépouillement dérivée de la méthode de Hesse et Rübartsch [18]. Le calcul est effectué sous contraintes (densité de probabilité $p(H) \geqslant 0)$ à l'aide de 51 sextuplets. Cette méthode présente l'avantage de supprimer les oscillations engendrées par la méthode de Hesse et Rübartsch sans contraintes. Comme nous l'avons discuté en détail dans [18], les résultats sont pratiquement insensibles au choix du paramètre de lissage entre $10^{-2}$ et $\sim 10$. Les calculs effectués avec un paramètre de lissage nul suggèrent que les distributions expérimentales sont de nature continue. Au-delà de $\sim 10$, les distributions calculées sont trop fortement lissées comme le montre le paramètre d'optimisation $X_{\lambda}$ [18]. 
La forme de raie utilisée dans ce calcul est déduite de l'hypothèse d'une répartition aléatoire des directions du champ hyperfin par rapport aux axes principaux du tenseur gradient de champ électrique. Elle est calculée au premier ordre en perturbation et dépend de l'effet quadrupolaire $\Delta$ et du paramètre d'asymétrie $\eta$. La raie, notée $S^{\eta}(\Delta)$, est symétrique pour $\eta=1$ et dissymétrique dans les autres cas [18].

Les effets quadrupolaires $\Delta$, nécessaires pour le calcul des spectres dans l'état magnétique, sont déduits de spectres enregistrés dans l'état paramagnétique avant que commence la cristallisation. Ces spectres sont en général faiblement dissymétriques [17] et traduisent l'existence de distributions d'effets quadrupolaires et de déplacements isomériques. Les valeurs des effets quadrupolaires moyens $\bar{\Delta}$ sont de l'ordre de $0,5 \mathrm{~mm} \mathrm{~s}^{-1}$ dans les alliages amorphes $\mathrm{Fe}-\mathrm{B},(\mathrm{Fe}-\mathrm{Ni}) \mathrm{PB},(\mathrm{Fe}-\mathrm{Ni}) \mathrm{SiB}$ [8]. Ils sont comparables aux effets quadrupolaires moyens mesurés dans les borures $\mathrm{FeB}[6], \mathrm{Co}_{3} \mathrm{~B}$ ou dans les carbures de fer. Dans ces composés cristallins, il n'existe aucune corrélation entre $H$ et $\Delta$. Comme une telle corrélation est $a$ priori difficile à imaginer, nous calculons la forme de raie en remplaçant la distribution d'effet quadrupolaire par l'effet quadrupolaire moyen $\bar{\Delta}$ avec $\eta$. Cette hypothèse sur $\eta$ permet d'éviter le problème, peut-être dénué de sens [17, Varret et Henry], du signe de l'effet quadrupolaire. De plus, au premier ordre pour $\Delta \lesssim 0,6 \mathrm{~mm} \mathrm{~s}^{-1}$

$$
\mathrm{S}^{1}(\Delta) \simeq \frac{1}{2}\left[\mathrm{~S}^{\eta}(\Delta)+\mathrm{S}^{\eta}(-\Delta)\right]
$$

quel que soit $\eta$. L'écart relatif entre le premier et le second membre de la relation (1) est au maximum de $9,8 \%$ (pour $\eta=0,|\Delta|=0,6 \mathrm{~mm} \mathrm{~s}^{-1}$ et pour une vitesse $\pm 1,2 \Gamma$ par rapport au maximum du pic). Ainsi, dans le cas où il existe autant de gradients positifs que de gradients négatifs [17] la valeur $\eta=1$ permet de bien approcher la forme de raie, au premier ordre, pour une valeur donnée de $|\Delta|$ et quelle que soit la distribution de $\eta=(1)$. Enfin, c'est la valeur $\eta=1$ qui conduit aux meilleurs ajustements des spectres expérimentaux (Fig. 2).

La figure 2 montre également que les spectres sont dissymétriques. L'amplitude des pics 2 et 3 est respectivement plus grande que celle des pics 5 et 4 (les pics sont numérotés de 1 à 6 des vitesses négatives aux vitesses positives). Cette dissymétrie peut résulter, entre autres :

1) De l'influence des effets quadrupolaires sur la forme de raie à condition qu'ils aient un signe déterminé et qu'ils soient suffisamment élevés pour que la théorie des perturbations au premier ordre cesse d'être valide. Comme nous l'avons déjà souligné, il est actuellement difficile d'estimer cet effet.

2) D'une corrélation entre le déplacement isomérique $\delta$ et le champ hyperfin $H$. Dans les alliages amorphes et les composés cristallins Fe-B, les déplacements isomériques moyens décroissent linéairement
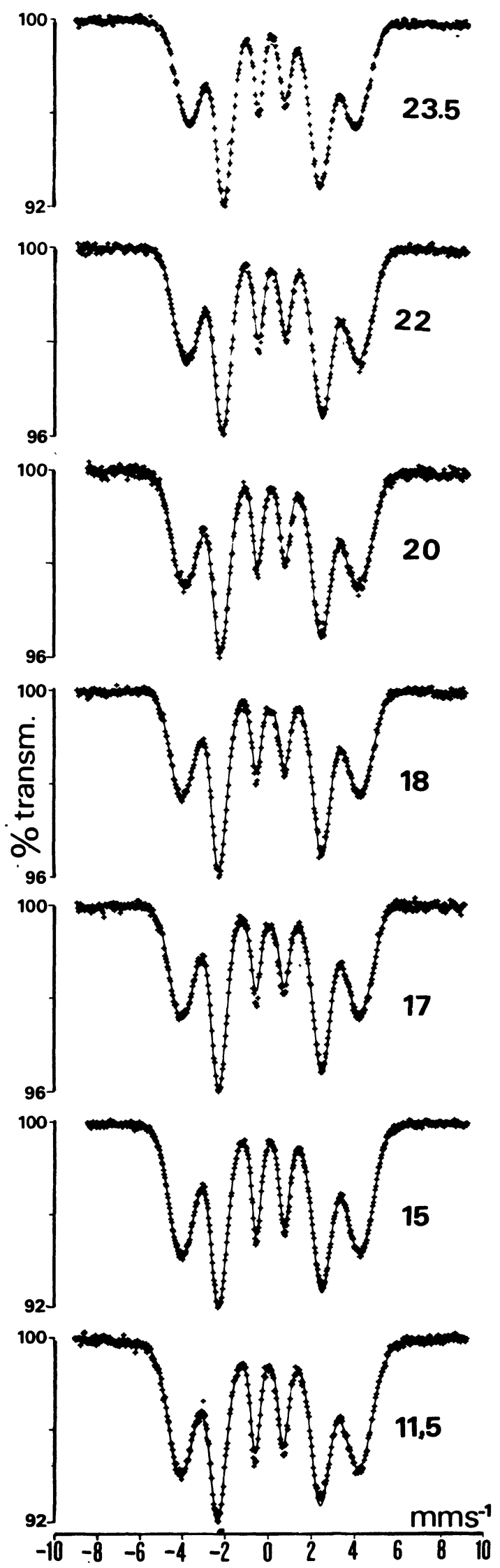

Fig. 2. - Spectres expérimentaux et calculés des alliages de composition $\mathrm{Fe}_{97-x} \mathrm{Si}_{3} \mathrm{~B}_{\mathbf{x}}$ à la température ambiante.

[Experimental and calculated spectra of $\mathrm{Fe}_{97-x} \mathrm{Si}_{3} \mathrm{~B}_{x}$ alloys at room temperature.] 
quand le champ hyperfin augmente $(\delta=a+b H$, $\left.b=-11 \times 10^{-4} \mathrm{~mm} \mathrm{~s}^{-1} \mathrm{kOe}^{-1}[6]\right)$. Ce signe de $b$ conduit cependant à une dissymétrie opposée à celle qui est observée (Fig. 2). Les déplacements isomériques mesurés dans le composé $\mathrm{Fe}_{3} \mathrm{~B}$ tétragonal n'obéissent pas à la relation précédente car ils décroissent puis croissent quand le champ hyperfin augmente.

3) De l'influence de champs dipolaires $H_{\mathrm{d}}$. Cet effet peut être estimé dans $\mathrm{Fe}_{80} \mathrm{~B}_{20}$ à l'aide des largeurs des six enveloppes déterminées par Price et al. [20] (Tableau I), en supposant l'identité des angles polaires dans les relations qui traduisent, au premier ordre, l'influence de l'effet quadrupolaire et du champ dipolaire.

Une formule approchée [18] permet de calculer les largeurs de chaque enveloppe du spectre en convoluant les effets dus à la distribution de champ hyperfin de largeur à mi-hauteur $\Delta H$ (terme $\left|\alpha_{j}\right| \Delta H$ (2)) et ceux dus à l'effet quadrupolaire et au champ dipolaire

$\Gamma_{j} \simeq 0,8 \Gamma+0,48\left|\beta_{j} \bar{\Delta}+2 \alpha_{j} H_{\mathrm{d}}\right|+\left|\alpha_{j}\right| \Delta H$

$\beta_{j}=1 \quad j=1,6 \quad \beta_{j}=-1 \quad j=2, \ldots, 5$

$\Gamma$ est la largeur de raie caractéristique du ${ }^{57} \mathrm{Fe}\left(\simeq 0,25 \mathrm{~mm} \mathrm{~s}^{-1}\right)$. Pour $\mathrm{Fe}_{80} \mathrm{~B}_{20},|\Delta|$ vaut $0,45 \mathrm{~mm} \mathrm{~s}^{-1}$ [8] et nos calculs donnent $\Delta H=75 \mathrm{kOe}$ (ou $80 \mathrm{kOe}$ [20]). La relation (2) permet de calculer (pour $2\left|\alpha_{j} H_{\mathrm{d}}\right|<|\bar{\Delta}|$, soit $H_{\mathrm{d}} \leqslant 14 \mathrm{kOe}$ ).

$\bar{\Gamma}_{k}=\left(\Gamma_{k}+\Gamma_{7-k}\right) / 2=0,8 \Gamma+0,48|\bar{\Delta}|+\left|\alpha_{k}\right| \Delta H$

$k=1,2,3$

Tableau I. - Largeurs expérimentales [20] et calculées (2) dans $\mathrm{Fe}_{80} \mathrm{~B}_{20}$.

[Experimental [20] and calculated (2) widths in $\left.\mathrm{Fe}_{80} \mathrm{~B}_{20} \cdot\right]$

$\Gamma_{j} \mathrm{~mm} \mathrm{~s}^{-1}$ exp.

$\begin{array}{lllllll}(j=1, \ldots, 6) & 1,67 & 1,10 & 0,57 & 0,63 & 1,21 & 1,57\end{array}$

$\begin{array}{lllllll}\Gamma_{j} \mathrm{~mm} \mathrm{~s}^{-1} \text { calc. } & 1,67 & 1,10 & 0,61 & 0,62 & 1,15 & 1,57\end{array}$

et de déduire des valeurs expérimentales du tableau I, $\Delta H=75 \mathrm{kOe}$ et $\Gamma=0,26 \mathrm{~mm} \mathrm{~s}^{-1}$, en bon accord avec nos résultats. La valeur $H_{\mathrm{d}}=3 \mathrm{kOe}$ conduit aux largeurs calculées du tableau $I$.

Le champ dipolaire nécessaire pour rendre compte de la dissymétrie du spectre est donc physiquement raisonnable. Cependant, la valeur $H_{\mathrm{d}}=3 \mathrm{kOe}$ ne constitue qu'un ordre de grandeur compte tenu des approximations faites dans (2). Des champs dipolaires de cet ordre sont observés dans $\mathrm{Fe}_{2} \mathrm{~B}$ [19] et peuvent, peut-être, expliquer les anomalies du spectre de $\mathrm{Fe}_{3} \mathrm{~B}$ tétragonal (voir Tableau II).

Le calcul précédent montre qu'il est inutile d'introduire une corrélation, difficile à justifier, entre $H$ et $\Delta$ ainsi que l'hypothèse $\eta=0$ [20] pour rendre compte des dissymétries des spectres. La discussion précédente peut également s'appliquer aux spectres de
$\mathrm{Fe}_{78} \mathrm{Si}_{10} \mathrm{~B}_{12}$ sous champ appliqué [22] $\left(\Gamma_{1}>\Gamma_{6}\right.$, $\left.\Gamma_{3}<\Gamma_{4}\right)$ et de $\mathrm{Fe}_{40} \mathrm{Ni}_{40} \mathrm{~B}_{20}$ [18]. Notons enfin que l'expression (2) conduit à une différence $d_{k}=\left|\Gamma_{k}-\Gamma_{7-k}\right|$ qui décroît de l'extérieur vers le centre du spectre alors que le tableau I montre que $d_{1} \simeq d_{2}$. La superposition des effets d'une corrélation $\delta-H$ à pente positive et de champs dipolaires permet d'obtenir un tel résultat.

Pour simuler les dissymétries des spectres expérimentaux, nous avons simplement introduit une relation linéaire entre $\delta$ et $H$ de pente $+6 \times 10^{-4} \mathrm{~mm} \mathrm{~s}^{-1} \mathrm{kOe}^{-1}$. Cette pente est comparable à celle qui a été utilisée par Takacs [23] dans les alliages amorphes $\mathrm{Fe}-\mathrm{B}$. Elle n'a pas nécessairement une signification physique (discussion précédente) et constitue un paramètre du dépouillement dont l'influence sur la distribution de champ calculée est faible.

La figure 2 présente les spectres expérimentaux (à $300 \mathrm{~K}$ ) d'alliages $\mathrm{Fe}_{97-x} \mathrm{Si}_{3} \mathrm{~B}_{x}$ qui ont été ainsi calculés. L'amplitude des transitions $\Delta m=0$, optimisée par la méthode de Fibonacci, varie peu avec $x$. Le rapport $A_{2} / A_{1}$ augmente de 0,85 à $\sim 1,10$ entre $x=11$ et $x=17$, puis reste sensiblement constant pour $x \geqslant 17$. Les distributions de champ hyperfin obtenues à $300 \mathrm{~K}$ dans les alliages $\mathrm{Fe}_{100-x} \mathrm{~B}_{x}(14 \leqslant x \leqslant 25)$ [8] sont reproduites sur la partie gauche de la figure 3 . Elles montrent une structure en 4 ou 5 pics distants de 25 à 30 kOe. Comme nous l'avons discuté en détail dans [18], cette structure résulte de la forme de raie et non de la méthode de calcul. La comparaison des champs hyperfins avec ceux qui ont été mesurés dans les borures cristallins conduit à attribuer ces pics aux atomes de fer ayant respectivement (en champ

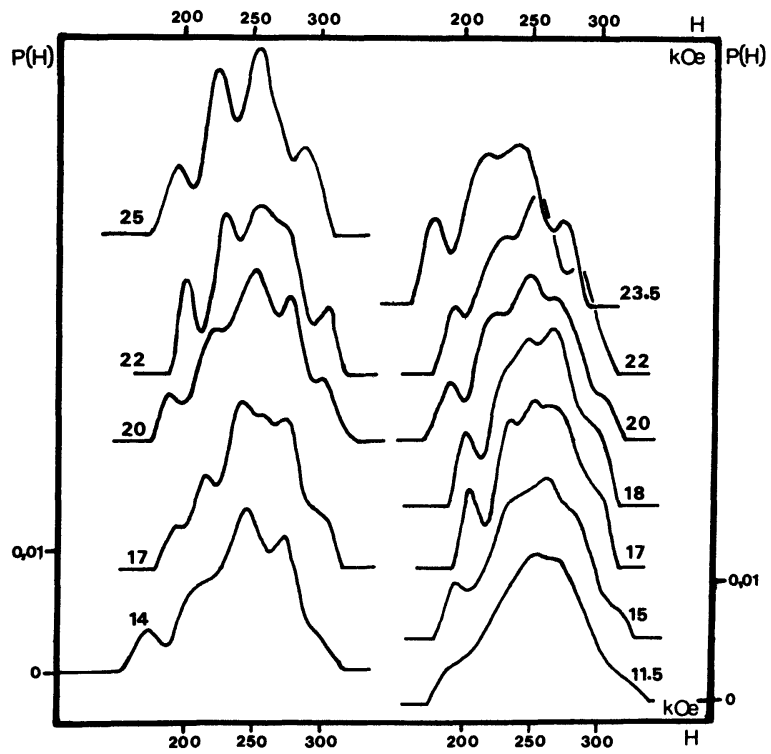

Fig. 3. - Distributions de champs hyperfins à la température ambiante des alliages $\mathrm{Fe}_{100-x} \mathrm{~B}_{x}$ (colonne de gauche [8]) et $\mathrm{Fe}_{97-x} \mathrm{Si}_{3} \mathrm{~B}_{x}$ (colonne de droite).

[Hyperfine field distributions at room temperature of $\mathrm{Fe}_{100-x} \mathrm{~B}_{x}$ (left-hand column [8]) and $\mathrm{Fe}_{97-x} \mathrm{Si}_{3} \mathrm{~B}_{x}$ alloys (right-hand column).] 
croissant) 5, 4, 3, 2 (ou 4, 3, 2, 1, 0) atomes de bore plus proches voisins. Le nombre moyen $\bar{n}_{\mathrm{B}}$ d'atomes de bore proches voisins d'un atome de fer varie alors de $\simeq 3(x=25)$ à $\simeq 1,6(x=14)$. Si l'on suppose qu'un atome de bore n'a pas d'atome de bore proche voisin, le décompte des paires $\mathrm{B}-\mathrm{Fe}$ et $\mathrm{Fe}-\mathrm{B}$ conduit à $(100-x) \bar{n}_{\mathrm{B}}=x \bar{Z}$ où $\bar{Z}$ est la coordinence moyenne des atomes de bore. On en déduit que le nombre de paires B-Fe reste voisin de 9 lorsque $x$ varie. Cette valeur s'accorde avec l'existence d'un motif structural commun aux alliages amorphes et aux borures cristallins. Ce motif est un polyèdre constitué d'un prisme trigonal d'atomes métalliques centré par le métalloïde et par trois atomes métalliques supplémentaires qui forment trois demi-octaèdres avec les faces rectangulaires du prisme. Ce polyèdre constitue l'unité de base du modèle structural de Gaskell [4] et du modèle quasi cristallin de Vincze $[7,11]$. Ce dernier modèle suppose que l'ordre local dans $\mathrm{Fe}_{75} \mathrm{~B}_{25}$ amorphe est semblable à celui du borure tétragonal $\mathrm{Fe}_{3} \mathrm{~B}$. Lorsque $x$ diminue, les atomes de fer se substituent aux atomes de bore au centre des prismes. Nos résultats [8] ne s'accordent pas avec ce modèle.

La figure 3 (partie droite) présente également les distributions de champ hyperfin déduites des spectres de la figure 2. La comparaison entre les deux ensembles de courbes est justifiée car les températures de Curie et les aimantations à saturation à $300 \mathrm{~K}$ de ces deux séries d'alliages sont très voisines [24, 15]. Trois remarques se dégagent de cette comparaison :

a) Il existe une similitude entre les distributions des alliages $\mathrm{Fe}_{100-x} \mathrm{~B}_{x}$ et $\left(\mathrm{Fe}_{100-x^{\prime}-3} \mathrm{Si}_{3}\right) \mathrm{B}_{x^{\prime}}$ pour $x \simeq x^{\prime}$ (ex. : $x=14, x^{\prime}=15 ; x=20, x^{\prime}=20,22$; $\left.x=22,25, x^{\prime}=23,5\right)$.

b) Les composantes élémentaires sont élargies par la présence de silicium (Fig. 4).

Les remarques $a$ ) et $b$ ) montrent que l'addition de silicium ne modifie pas la structure des alliages amorphes $\mathrm{Fe}-\mathrm{B}$, au moins tant que la concentration en cet élément n'est pas trop importante. Les résultats précédents suggèrent que le silicium ne se substitue pas au bore mais qu'il occupe des sites semblables à ceux du fer. Dans le premier cas, de nouvelles composantes devraient apparaître sur les distributions de champ hyperfin. Dans le second cas, un élargissement analogue des différentes composantes de ces distributions est attendu. La figure 4 illustre un tel comportement pour les alliages $\mathrm{Fe}_{86} \mathrm{~B}_{14}, \mathrm{Fe}_{82} \mathrm{Si}_{3} \mathrm{~B}_{15}$, $\mathrm{Fe}_{76} \mathrm{Si}_{9} \mathrm{~B}_{15}$ et montre que les distributions d'alliages à teneurs en bore voisines se comparent bien entre elles. Enfin, l'apparition d'une ferrite au silicium lors de la première étape de cristallisation (paragraphe 5.2) est en bon accord avec ce modèle.

5. Mécanismes de retour à l'équilibre. - Les mécanismes de cristallisation des alliages amorphes dépendent du système considéré et peuvent être résumés comme suit dans le cas des alliages $\mathrm{Fe}_{100-x} \mathrm{~B}_{x}$ [7] :

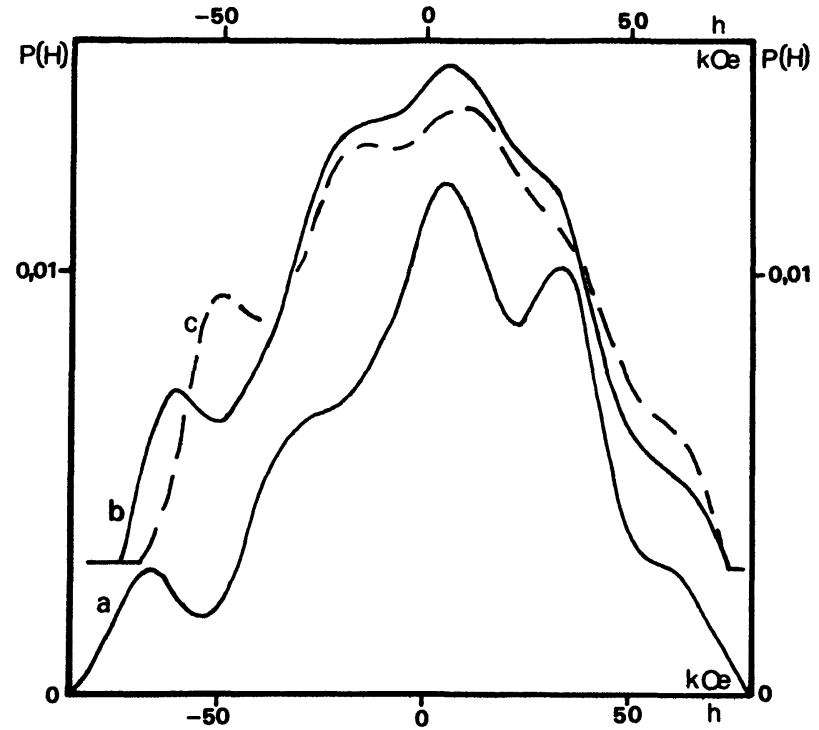

Fig. 4. - Comparaison entre les distributions de champs hyperfins à la température ambiante des alliages : a) $\mathrm{Fe}_{86} \mathbf{B}_{14}$ [8], b) $\left.\mathrm{Fe}_{82} \mathrm{Si}_{3} \mathrm{~B}_{15}, c\right) \mathrm{Fe}_{76} \mathrm{Si}_{9} \mathrm{~B}_{15}$. Les champs hyperfins moyens sont superposés $h=H-\bar{H}$.

[Comparison of hyperfine field distributions at room temperature of : a) $\mathrm{Fe}_{86} \mathrm{~B}_{14}[8]$, b) $\mathrm{Fe}_{82} \mathrm{Si}_{3} \mathrm{~B}_{15}$, c) $\mathrm{Fe}_{76} \mathrm{Si}_{9} \mathrm{~B}_{15}$. The mean hyperfine fields are superimposed $h=H-H$.]

1) $\mathrm{Fe}_{100-x} \mathrm{~B}_{x}$ amorphe $\rightarrow \mathrm{Fe}$ c.c. $+\mathrm{Fe}_{75} \mathrm{~B}_{25}$ amorphe,

2) $\mathrm{Fe}_{75} \mathrm{~B}_{25}$ amorphe $\rightarrow \mathrm{Fe}_{3} \mathrm{~B} \varepsilon_{1}$ tétragonal,

3) $\mathrm{Fe}_{3} \mathrm{~B} \varepsilon_{1} \rightarrow \mathrm{Fe}$ c.c. $+\mathrm{Fe}_{2} \mathrm{~B}$.

Les deux premières étapes sont séparées pour $x \leqslant 17$ alors qu'elles se recouvrent pour $x>17$. La décomposition du borure $\mathrm{Fe}_{3} \mathrm{~B} \varepsilon_{1}$ métastable occupe un large domaine de température et ne semble intervenir qu'au-delà de $825 \mathrm{~K}$ [21].

La figure 5 présente l'évolution avec la température de la résistance ohmique réduite d'un échantillon amorphe de composition $\mathrm{Fe}_{82} \mathrm{Si}_{3} \mathrm{~B}_{15}$. Cette courbe montre en particulier deux étapes de cristallisation de la phase amorphe repérées par les températures $T_{\mathrm{cr}_{1}}$ et $T_{\mathrm{cr}_{2}}$ dont les variations avec la composition sont comparées à celles des alliages $\mathrm{Fe}_{100-x} \mathrm{~B}_{x}$ sur la figure 7 [24]. Afin de préciser les éventuels changements de l'ordre local et les phases qui apparaissent lors des transformations, nous avons utilisé un four à faible inertie thermique, transparent aux rayons $\gamma$ [25]. Les différentes étapes du retour à l'équilibre sont repérées par résistivité électrique et les transformations sont arrêtées par trempe rapide. On enregistre alors à la température ambiante un spectre Mössbauer caractérisant un état proche de celui obtenu en cours de transformation. Les spectres a à $f$ de la figure 5 se rapportent tous au même échantillon, qui a subi des traitements thermiques cumulatifs réalisés avec une vitesse de chauffage de $10 \mathrm{~K} \mathrm{~min}^{-1}$. On vérifie bien dans l'état amorphe, que le comportement de la résistivité est partiellement réversible. Pour simplifier la figure 5, nous n'avons pas porté les parties de courbes correspondant aux différents 


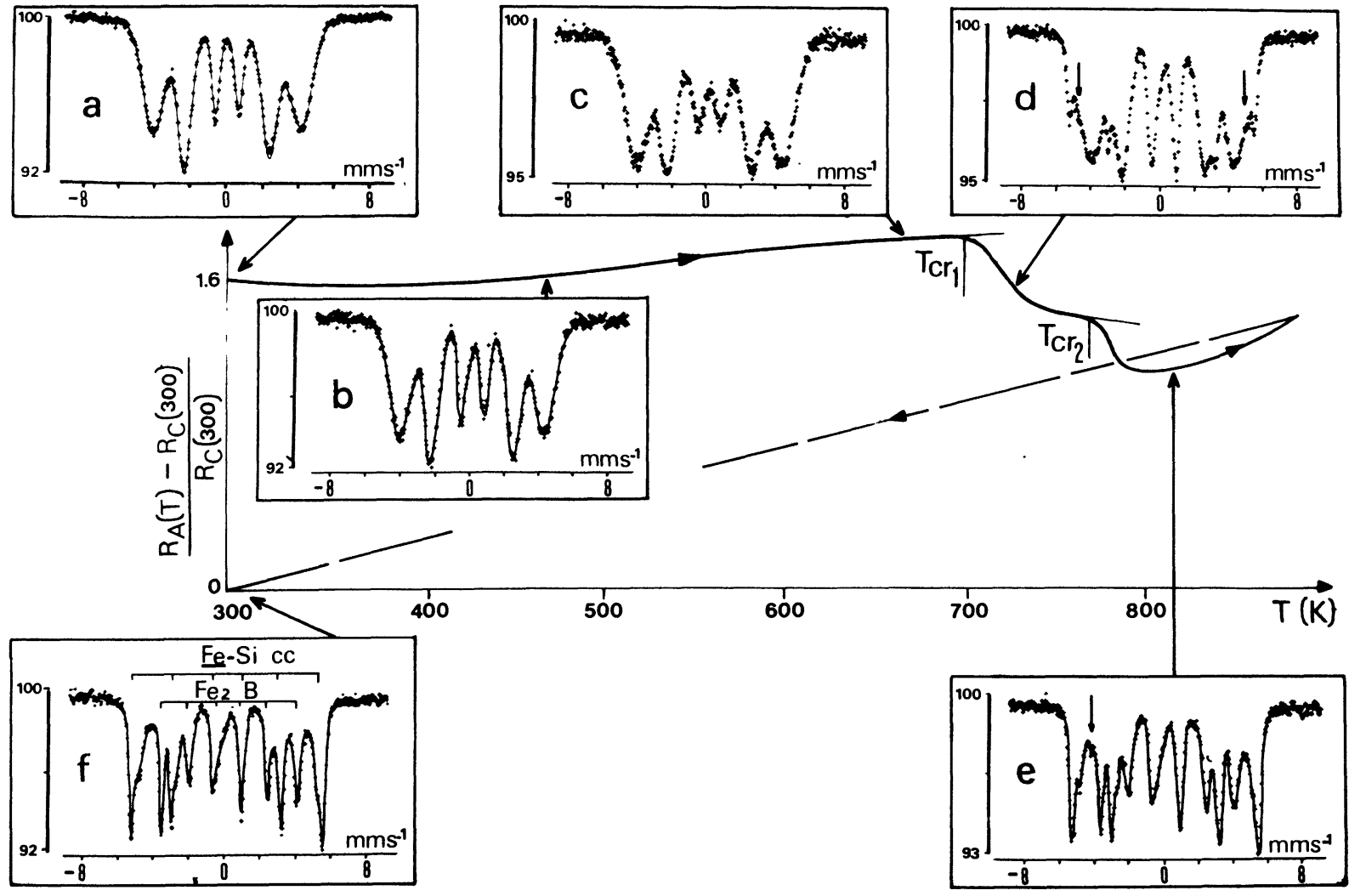

Fig. 5. - Variation de la résistance électrique réduite avec la température (vitesse de chauffage $10 \mathrm{~K}$ min. ${ }^{-1}$ ). Les spectres Mössbauer à la température ambiante ont été enregistrés après que l'échantillon ait atteint la température indiquée par la flèche et qu'il ait été trempé (vitesse de refroidissement $15 \mathrm{~K} \mathrm{~s}^{-1}$ ).

[Variation of the reduced electrical resistance versus temperature (heating rate $10 \mathrm{~K} \mathrm{~min}^{-1}$ ). The room temperature Mössbauer spectra have been recorded after heating the sample up to the temperature shown by an arrow and quenching it to room temperature (cooling rate $\left.15 \mathrm{~K} \mathrm{~s}^{-1}\right)$.]

traitements. Les données déduites de l'étude par résistivité seront publiées ultérieurement.

5.1 Relaxation Structurale. - La relaxation structurale se traduit sur la figure 5 par des écarts à la linéarité de la résistivité en fonction de la température. Les spectres $\mathrm{a}, \mathrm{b}$ et $\mathrm{c}$ de la figure 5 présentent une diminution du rapport $A_{2} / A_{1}$ des intensités des raies 2 et 5 à celles des raies 1 à 6 . Les spectres Mössbauer d'un échantillon de composition $\mathrm{Fe}_{76} \mathrm{Si}_{9} \mathrm{~B}_{15}$ enregistrés à $300 \mathrm{~K}$ après des recuits isothermes à $723 \mathrm{~K}$ montrent une variation semblable du rapport $A_{2} / A_{1}$ (Fig. 6). On observe donc une rotation de l'induction magnétique d'une direction proche du plan du ruban à une position voisine de la normale à ce plan. Ce phénomène a été mis en évidence dans divers autres systèmes $[26,27]$ et est attribué à la relaxation des contraintes résiduelles introduites par la trempe depuis l'état liquide. Il pourrait être accompagné par la création de nouveaux domaines magnétiques [27].

Les distributions de champ hyperfin déduites des spectres a et $b$ de la figure 5 , ainsi que d'un spectre intermédiaire, ne présentent pas de différences significatives. Il semblerait donc que l'ordre local n'ait pas été affecté par les traitements thermiques cor-

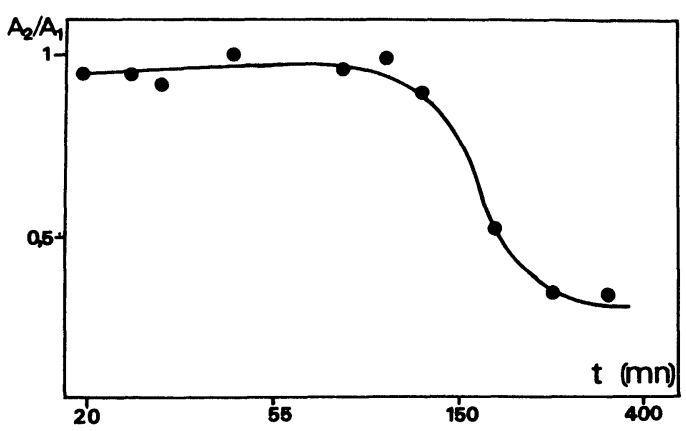

Fig. 6. - Variation du rapport des intensités $A_{2} / A_{1}$ avec la durée de revenu à $723 \mathrm{~K}$.

[Variation of the intensity ratio $A_{2} / A_{1}$ as a function of the annealing time at $723 \mathrm{~K}$.]

respondants. Par contre, la forme du spectre c diffère des précédentes. On remarque un changement de l'enveloppe des pics externes et surtout un élargissement de près de $100 \%$ des raies internes. Une distribution de champs hyperfins calculée dans les hypothèses du paragraphe 4 ne rend pas correctement compte du spectre, en particulier au voisinage de la vitesse zéro. Comme ce spectre a été enregistré après une montée en température jusqu'au voisinage de la 
température de transition vitreuse $\left(T_{\mathrm{g}}=760 \mathrm{~K}\right.$, S. Al Bijat et al., à paraître), il est possible qu'un changement d'ordre local soit intervenu. De nouvelles expériences sont en cours pour préciser ce point.

5.2 Cristallisation. - Nous nous sommes intéressés à la cristallisation de deux alliages de composition $\mathrm{Fe}_{82} \mathrm{Si}_{3} \mathrm{~B}_{15}$ et $\mathrm{Fe}_{76} \mathrm{Si}_{9} \mathrm{~B}_{15}$ qui présentent, d'après les variations de résistivité avec la température, respectivement deux étapes et une étape de cristallisation de la phase amorphe [S. Al Bijat et al., à paraître]. Les températures de début de cristallisation du premier alliage sont supérieures de $100 \mathrm{~K}$ environ aux températures correspondantes mesurées dans l'alliage $\mathrm{Fe}_{85} \mathrm{~B}_{15}$. Dans le cas de l'alliage $\mathrm{Fe}_{76} \mathrm{Si}_{9} \mathrm{~B}_{15}$, la mesure de la résistivité ne met en évidence qu'une seule température de cristallisation, $T_{\mathrm{cr}}=840 \mathrm{~K}$. Les spectres Mössbauer permettent d'identifier les phases qui apparaissent au cours de la cristallisation. Les fractions de fer dans chaque phase résultent d'un dépouillement à l'aide d'un programme qui optimise les aires relatives de spectres de composés cristallins donnés a priori. Les paramètres hyperfins des solutions solides Fe-Si c.c., des borures $\mathrm{Fe}_{2} \mathrm{~B}$, $\mathrm{Fe}_{3} \mathrm{~B}$ orthorhombique et tétragonal, $\mathrm{Fe}_{5} \mathrm{SiB}_{2}$ ont été utilisés dans le dépouillement [28, 19, 29, Tableau II, 30 ] et les facteurs Debye-Waller de ces phases ont été supposés égaux.

Un dépouillement en trois sites de fer du spectre Mössbauer de $\mathrm{Fe}_{3} \mathrm{~B} \varepsilon_{1}$ a été publié par Chien et al. [6]. Contrairement aux conclusions de ces auteurs, ces résultats montrent que ce borure n'est pas isomorphe du phosphure $\mathrm{Fe}_{3} \mathrm{P} \varepsilon$ mais du phospho-borure $\left(\mathrm{Fe}_{3}(\mathrm{P}, \mathrm{B}) \varepsilon_{1}\right)$. Un excellent accord existe en effet entre les paramètres hyperfins de $\mathrm{Fe}_{3} \mathrm{~B}$ et ceux publiés pour le composé $\mathrm{Fe}_{2,88} \mathrm{P}_{0,05} \mathrm{~B}_{0,95} \varepsilon_{1}$ par Wäppling et al. [31] (Tableau II). Par contre, les composés $\varepsilon$ et $\varepsilon_{1}$ présentent le même ordre local [32] et il est possible, comme le soulignent Chien et al. [6], que chaque site de $\mathrm{Fe}_{3} \mathrm{~B} \varepsilon_{1}$ donne naissance à deux sextuplets comme c'est le cas dans $\mathrm{Fe}_{3} \mathrm{P} \varepsilon$ [33]. Un dépouillement en six sextuplets d'égales intensités améliore en effet le $\chi^{2}$ et conduit à des largeurs de raies raisonnables. Les paramètres hyperfins déduits de ce calcul sont présentés dans le tableau II. L'écart entre les champs hyperfins des atomes de fer d'un même site cristallographique pourrait être lié à l'existence d'une structure magnétique complexe et de champs dipolaires de $\sim 6-9 \mathrm{kOe}$.

Les résultats des dépouillements des spectres se résument de la façon suivante :

5.2.1 Alliage $\mathrm{Fe}_{82} \mathrm{Si}_{3} \mathrm{~B}_{15}$, vitesse de chauffage $10 \mathrm{~min}^{-1}$ (Fig. 5).

- Première étape : $T_{\mathrm{cr}_{1}}<T<T_{\mathrm{cr}_{2}}$. Le spectre d (Fig. 5) montre l'apparition d'une ferrite au silicium (pics repérés par une flèche) au sein de la matrice amorphe. Aucun borure n'est détecté.

- Deuxième étape : $T \gtrsim T_{\mathrm{cr}_{2}}$ (spectre e, Fig. 5). La phase amorphe a disparu. On distingue nettement une ferrite au silicium de composition voisine de $\mathrm{Fe}-6 \% \mathrm{Si}$ et le borure stable $\mathrm{Fe}_{2} \mathrm{~B}$. Le pic marqué d'une flèche pourrait provenir d'une très faible quantité $(<2 \%)$ de borure métastable $\mathrm{Fe}_{3} \mathrm{~B} \varepsilon_{1}$. L'équilibre complet de l'alliage est obtenu après la mise en ordre de la ferrite au silicium (spectre f, Fig. 5), qui explique le comportement de la résistivité au-delà de $T_{\text {cr }_{2}}$. La fraction de fer dans le borure $\mathrm{Fe}_{2} \mathrm{~B}$ augmente très peu $(2 \%$ environ). Ce faible accroissement pourrait provenir de la décomposition du borure $\mathrm{Fe}_{3} \mathrm{~B} \varepsilon_{1}$. Enfin, le calcul montre que $36,5 \%$ des atomes de fer se trouvent dans $\mathrm{Fe}_{2} \mathrm{~B}$, en parfait accord avec la teneur initiale en bore de l'alliage.

5.2.2 Alliage $\mathrm{Fe}_{76} \mathrm{Si}_{9} \mathrm{~B}_{15}$, recuit isotherme à $723 \mathrm{~K}$. - La figure 8 résume les résultats obtenus et montre que la cristallisation se déroule en deux étapes au cours d'un recuit isotherme. Le borure stable $\mathrm{Fe}_{2} \mathrm{~B}$ et une ferrite au $\mathrm{Si}$ apparaissent dès la première étape de cristallisation comme le prouvent (Fig. 9), les pics étroits qui se superposent aux raies larges de la phase amorphe résiduelle. On ne met pas en évidence la présence de borures métastables ou du composé $\mathrm{Fe}_{5} \mathrm{SiB}_{2}$.

$\mathrm{Au}$ cours de la première étape, les largeurs de raies de la phase amorphe résiduelle diminuent et ses paramètres hyperfins évoluent.

Un bilan des éléments contenus dans les phases cristallines permet de montrer que la phase amorphe s'appauvrit en silicium et s'enrichit en bore. Les incertitudes de cette détermination permettent seule-

Tableau II. - Paramètres hyperfins du borure $\mathrm{Fe}_{3} \mathrm{~B} \varepsilon_{1}$ à la température ambiante.

[Room temperature hyperfine parameters of $\mathrm{Fe}_{3} \mathrm{~B} \varepsilon_{1}$.]

\begin{tabular}{|c|c|c|c|c|c|c|}
\hline \multicolumn{2}{|c|}{ Site } & $\begin{array}{c}\mathrm{Fe}_{2,88} \mathrm{P}_{0,05} \mathrm{~B}_{0,95} \varepsilon_{1} \\
H \mathrm{kOe}[31]\end{array}$ & $H \mathrm{kOe}$ & $\Delta=2 \varepsilon \mathrm{mm} \cdot \mathrm{s}^{-1}$ & $\delta \mathrm{mm} \cdot \mathrm{s}^{-1}$ & $\Gamma \mathrm{mm} \cdot \mathrm{s}^{-}$ \\
\hline & & - & - & - & - & - \\
\hline & Ia & $288,6 \pm 0,2$ & $293 \pm 1$ & $-0,09 \pm 0,03$ & $0,06 \pm 0,02$ & 0,39 \\
\hline 1 & $\mathrm{Ib}$ & & $281 \pm 1$ & $0,09 \pm 0,03$ & $0,06 \pm 0,02$ & 0,39 \\
\hline II & IIa & $229,7 \pm 0,1$ & $230 \pm 1$ & $-0,10 \pm 0,03$ & $0,10 \pm 0,02$ & 0,39 \\
\hline 11 & $\mathrm{IIb}$ & & $218 \pm 1$ & $-0,09 \pm 0,02$ & $0,10 \pm 0,02$ & 0,39 \\
\hline & IIIa & $263,8 \pm 0,2$ & $269 \pm 1$ & $0,08 \pm 0,02$ & $0,02 \pm 0,02$ & 0,39 \\
\hline III & IIIb & & $257 \pm 1$ & $0,18 \pm 0,02$ & $0,05 \pm 0,02$ & 0,39 \\
\hline
\end{tabular}


ment d'affirmer que la teneur en silicium est au plus de 2 at. $\%$ alors que la teneur en bore est au moins de 20 at. $\%$.

Nos résultats s'accordent avec ceux de Den Broeder et al. [34] mais diffèrent de ceux de Hoselitz [35] qui observe la présence simultanée d'une ferrite au silicium, du borure $\mathrm{Fe}_{3} \mathrm{~B}$ et d'une phase amorphe résiduelle entre $T_{\mathrm{cr}_{1}}$ et $T_{\mathrm{cr}_{2}}$ dans $\left(\mathrm{Fe}_{100-y} \mathrm{Si}_{y}\right)_{88} \mathrm{~B}_{12}$ $(y=9 ; 15)$. Les résultats de Hoselitz diffèrent d'ailleurs de ceux de Den Broeder et al. pour l'alliage $\mathrm{Fe}_{80} \mathrm{Si}_{8} \mathrm{~B}_{12}(y=9)$.

Deux faits essentiels distinguent donc la cristallisation des alliages $\mathrm{Fe}-\mathrm{Si}-\mathrm{B}$ de celle des alliages $\mathrm{Fe}-\mathrm{B}$ :

- Le silicium stabilise fortement la structure amorphe (Fig. 7).

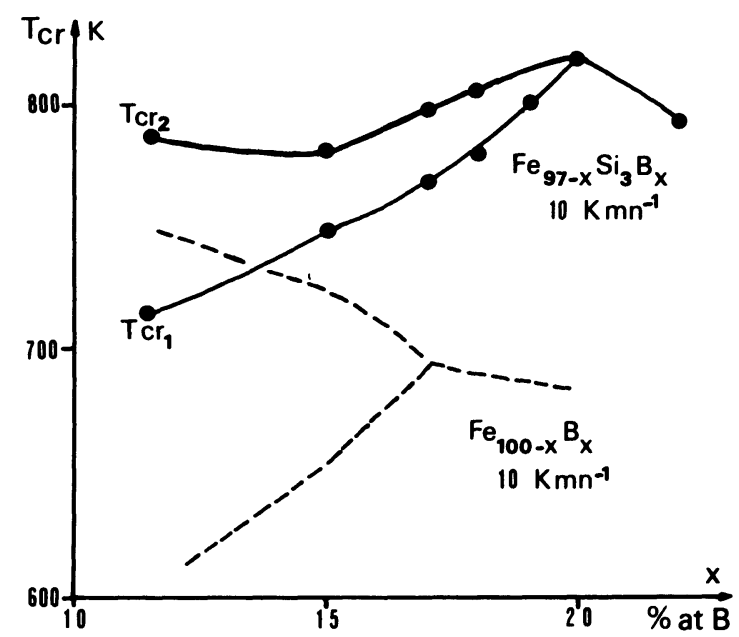

Fig. 7. - Températures de début de cristallisation en fonction de la teneur en bore des alliages $\mathrm{Fe}_{100-x}$ [24] et $\mathrm{Fe}_{97-x} \mathrm{Si}_{3} \mathrm{~B}_{x}$.

[Start of crystallisation temperatures versus boron content in $\mathrm{Fe}_{100-x} \mathrm{~B}_{x}$ [24] and $\mathrm{Fe}_{97-x} \mathrm{Si}_{3} \mathrm{~B}_{x}$ alloys.]

- La décomposition de la phase amorphe conduit directement aux phases stables.

Il est d'ailleurs intéressant de noter le rôle similaire $\mathrm{du}$ silicium dans la stabilisation de ces alliages amorphes et dans celle des phases $\varepsilon$ métastables $\mathrm{Fe}-\mathrm{C}$ et $\mathrm{Fe}-\mathrm{C}-\mathrm{Si}[13]$.

La localisation du bore dans des sites interstitiels stables rend cet élément peu mobile [36]. La figure 8 suggère également que l'enrichissement en bore et l'appauvrissement en silicium de la phase amorphe résiduelle se produisent essentiellement par la formation d'une ferrite au silicium alors que la fraction de fer dans $\mathrm{Fe}_{2} \mathrm{~B}$ reste pratiquement constante.

Le silicium et le fer seraient donc plus mobiles que le bore. Cette hypothèse est cohérente avec la description de la structure de ces alliages faite au paragraphe 4 et avec la réaction, amorphe $\rightarrow$ solution solide $\mathrm{Fe}-\mathrm{Si}+$ amorphe enrichi en bore, qui se produit entre $T_{\mathrm{cr}_{1}}$ et $T_{\mathrm{cr}_{2}}$. L'augmentation de $T_{\mathrm{cr}_{1}}$ par addition de silicium aux alliages $\mathrm{Fe}-\mathrm{B}$ pourrait provenir d'une diminution des coefficients de diffusion.

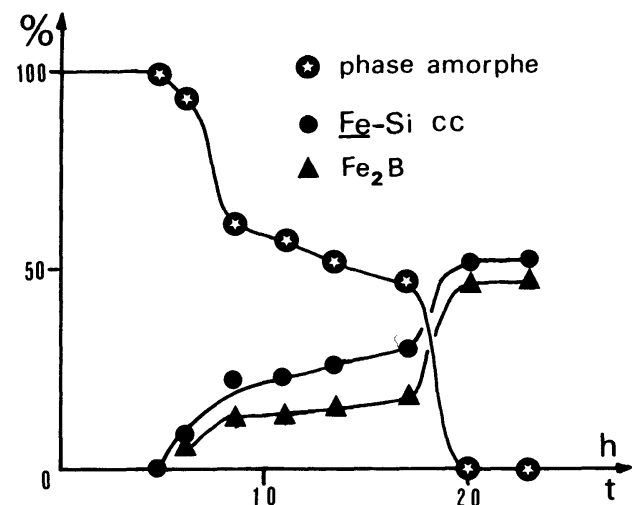

Fig. 8. - Evolution des proportions d'atomes de fer dans les différentes phases au cours de la cristallisation à $723 \mathrm{~K}$ de l'alliage $\mathrm{Fe}_{76} \mathrm{Si}_{9} \mathrm{~B}_{15}$.

[Evolution of the iron atom proportions in the various phases during the isothermal crystallisation at $723 \mathrm{~K}$ of the $\mathrm{Fe}_{76} \mathrm{Si}_{9} \mathrm{~B}_{15}$ alloy.]

L'obtention d'une quantité faible ou nulle de borure $\mathrm{Fe}_{3} \mathrm{~B} \varepsilon_{1}$ est plus difficile à interpréter. En effet, $T_{\mathrm{cr}_{2}}$ est inférieure à la température limite d'existence de ce borure. Ainsi, pour $\mathrm{Fe}_{82} \mathrm{Si}_{3} \mathrm{~B}_{15}, T_{\mathrm{cr}_{2}}=780 \mathrm{~K}$, alors que $\mathrm{Fe}_{3} \mathrm{~B}$ ne se décompose qu'après un maintien prolongé (> $10 \mathrm{~min}$.) à $825 \mathrm{~K}$ [6].

Le ralentissement de la diffusion et éventuellement une faible solubilité du silicium dans le borure $\mathrm{Fe}_{3} \mathrm{~B}$ pourraient expliquer un tel comportement. Le premier effet ralentirait l'enrichissement en bore de la phase amorphe et repousserait la formation de $\mathrm{Fe}_{3} \mathrm{~B}$ à une température trop élevée. L'insolubilité du silicium dans $\mathrm{Fe}_{3} \mathrm{~B}$ produirait des conséquences semblables en retardant la nucléation et en ralentissant la croissance de ce borure. Notons que le carbure $\varepsilon$ est stabilisé par le silicium qui est pratiquement insoluble dans la cémentite $\mathrm{Fe}_{3} \mathrm{C}$ [37] dont les motifs structuraux sont semblables à ceux de $\mathrm{Fe}_{3} \mathrm{~B}$.

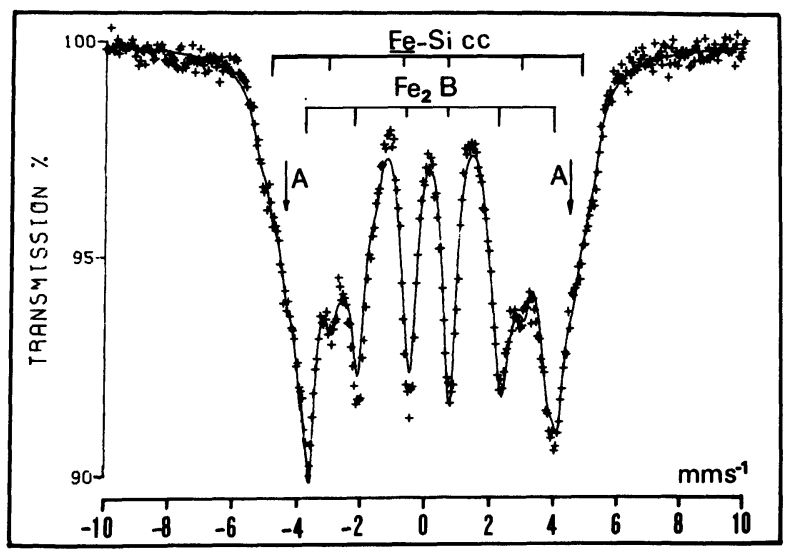

Fig. 9. - Spectre Mössbauer à la température ambiante de l'alliage $\mathrm{Fe}_{76} \mathrm{Si}_{9} \mathrm{~B}_{15}$ après un maintien de $12 \mathrm{~h}$ à $723 \mathrm{~K}$. Les pics externes de la phase amorphe sont indiqués par des flèches.

[Mössbauer spectrum at room temperature of the $\mathrm{Fe}_{76} \mathrm{Si}_{9} \mathrm{~B}_{15}$ alloy annealed $12 \mathrm{~h}$ at $723 \mathrm{~K}$. The external peaks of the amorphous phase are shown by arrows.] 
6. Conclusion. - Les spectres Mössbauer d'alliages $\mathrm{Fe}_{100-x-y} \mathrm{Si}_{y} \mathrm{~B}_{x}(11,5 \leqslant x \leqslant 23,5 y=3$ et 9$)$ bruts de trempe ont été analysés et les causes possibles des élargissements dissymétriques des raies (corrélation entre le champ hyperfin et le déplacement isomérique, champs dipolaires) ont été discutées. La comparaison des distributions de champ hyperfin des alliages $\mathrm{Fe}_{100-x} \mathrm{~B}_{x}$ et $\mathrm{Fe}_{100-x-y} \mathrm{Si}_{y} \mathrm{~B}_{x}$ montre que le silicium et le fer occupent des sites semblables. Le silicium augmente fortement la stabilité des alliages amorphes $\mathrm{Fe}-\mathrm{B}$. Il empêche la formation du borure métastable $\mathrm{Fe}_{3} \mathrm{~B} \varepsilon_{1}$ qui caractérise la deuxième étape de cristal- lisation des alliages $\mathrm{Fe}-\mathrm{B}$. C'est le borure $\mathrm{Fe}_{2} \mathrm{~B}$ qui se forme au cours de cette étape.

Remerciements. - Nous remercions le Professeur B. Roques (Laboratoire de Chimie du Solide Minéral, Université de Nancy I) pour les moyens d'élaboration des alliages de départ qu'il a mis à notre disposition et MM. S. Scherrer et S. Weber (Laboratoire de Physique du Solide, Ecole des Mines, Nancy) pour leur collaboration lors des dosages à l'analyseur ionique. Le présent travail a été financé par le C.N.R.S. (A.T.P. Matériaux Solides).

\section{Bibliographie}

[1] Rapidly Quenched Metals III, Ed. B. Cantor (The Metals Society), Vol. 1, 2 (1978).

[2] PolK, D. E., Acta Met. 20 (1972) 485.

[3] Boudreaux, D. S., Phys. Rev. B 18 (1978) 4039.

[4] Gaskell, P., J. Non-Cryst. Sol. 32 (1979) 207.

[5] Fukunaga, T., Misawa, M., Fukamichi, K., Masumoto, T., Suzuki, K., Rapidly Quenched Metals III (Ref. 1) 2 (1978) 325.

[6] Chien, C. L., Musser, D., Gyorgy, E. M., Sherwood, R. C., Chen, H. S., Luborsky, F. E., Walter, J. L., Phys. Rev. B 20 (1979) 283

[7] Kemeny, T., Vincze, I., Fogarassy, B., Arajs, S., Phys. Rev. B 20 (1979) 476

[8] Dubois, J. M., Le Caer, G., Amamou, A., Herold, U., Proceedings of the International Conference on the Applications of the Mössbauer Effect, Portoroz (1979), J. Physique Colloq. 41 (1980) C1-247.

[9] Wong, J., Lytle, F. W., Liebermann, H. H., Walter, J. L., LUBORSKY, F. E., Stanford Synchrotron Radiation Laboratory Activity Report (1979).

[10] Nold, E., SteEb, S., I.L.L., Rapport Annuel (1978).

[11] VincZe, I., Kemeny, T., ARAss, S., à paraître.

[12] Davies, H. A., Rapidly Quenched Metals III (Ref. 1) 1 (1978) 1.

[13] Dubois, J. M., Le Caer, G., Acta Met. 25 (1977) 609.

[14] Liebermann, H. H., Graham, C. D., I.E.E.E. Trans. on Mag. MAG 12 (1976) 921.

[15] Luborsky, F. E., Becker, J. J., Walter, J. L., LieberMANN, H. H., I.E.E., Trans. on Mag. MAG 15 (1979) 1146.

[16] Fujimori, H., Masumoto, T., Supplement to Science Reports of the Research Institutes, Tohoku University, A (1978) 181.

[17] Bletry, J., Dixmier, J., Dubois, J. M., Durand, J., Ferey, G., Henry, M., Janot, C., Le Caer, G., Mangin, P., Sadoc, J. F., Varret, F., Revue Phys. Appl. (1979) (ce numéro).

[18] Le Caer, G., Dubois, J. M., J. Phys. E : Sci. Instrum. 12 (1979) 1083.
[19] Weisman, I. D., Swartzenbruder, L. J., Bennett, L. H., Phys. Rev. 177 (1969) 465.

[20] Price, D. C., Campbell, S. J., Back, P. J., Proceedings of the International Conference on the Applications of the Mössbauer Effect. Portoroz (1979) J. Physique Colloq. 41 (1980) C1-263.

[21] ChIEN, C. L., Phys. Rev. B 18 (1978) 1003.

[22] Schurer, P. J., Morrish, A. H., Solid State Commun. 28 (1978) 819.

[23] TaKacs, L., Proceedings of the International Conference on the Applications of the Mössbauer Effect, Portoroz (1979) J. Physique Colloq. 41 (1980) C1-265.

[24] Luborsky, F. E., LiebermanN, H. H., Becker, J. J., WALTER, J. L., Rapidly Quenched Metals III, Ed. B. Cantor (the Metals Society) 2 (1978) 188.

[25] Dubois, J. M., Le Caer, G., J. Phys. E : Sci. Instrum. (1979) Colloq. 41 (1980) C1-247.

[26] Brown, D. K., Nowik, I., Paul, D. I., Solid State Commun. 24 (1977) 711.

[27] Tseng, P. K., Chuang, S. Y., Chen, H. S., J. Appl. Phys. 50 (1979) 4292.

[28] Haggstrom, L., Granas, L., Wappling, R., Devanaraya- NAN, S., Phys. Script. 7 (1973) 125.

[29] Choo, W. K., Kaplow, R., Met. Trans. A 8 (1977) 417.

[30] Wäppling, R., Ericsson, T., Haggstrom, L., ANDerson, Y., J. Physique Colloq. 37 (1976) C6-591.

[31] Wäppling, R., HaGgStrom, L., RundQvist, S., Karlsson, E., J. Solid State Chem. 3 (1971) 276.

[32] Rundevist, S., Acta Chem. Scan. 16 (1962) 1.

[33] Lisher, E. J., Wilkinson, C., Ericsson, T., Haggstrom, L., Lundgren, L., WäpPling, R., J. Phys. C : Sol. St. Phys. 7 (1974) 1344

[34] Den Broeder, F. J. A., Van der Borst, J., J. Appl. Phys. 50 (1979) 4279.

[35] Hoselitz, K., Phys. Status Solidi (a) 53 (1979) K 23.

[36] Suzuki, H. G., Y амамото, K., Mater. Sci. Eng. 33 (1978) 57.

[37] Lorenzo, A., Simon, A., C.R. Hebd. Séan. Acad. Sci. 275C (1973) 157. 